\title{
Propiedades psicométricas de la Escala de Autoeficacia Percibida Específica de Situaciones Académicas en una muestra de estudiantes españoles de Educación Secundaria Obligatoria
}

\author{
José M. García-Fernández ${ }^{1}$, Cándido J. Inglés ${ }^{2}$, María S. Torregrosa ${ }^{2}$, Cecilia \\ Ruiz-Esteban ${ }^{3}$, Ángela Díaz-Herrero ${ }^{3}$, Elena Pérez-Fernández ${ }^{2}$ y \\ María C. Martínez-Monteagudo ${ }^{2}$ \\ ${ }^{1}$ Universidad de Alicante, ${ }^{2}$ Universidad Miguel Hernández de Elche, \\ ${ }^{3}$ Universidad de Murcia (España)
}

\begin{abstract}
El objetivo de este estudio consistió en analizar las propiedades psicométricas de la Escala de Autoeficacia Percibida Específica de Situaciones Académicas en una muestra de 656 estudiantes españoles de Educación Secundaria Obligatoria de 12 a 16 años. Los análisis factoriales revelaron que la EAPESA presenta una estructura unidimensional, con coeficientes de consistencia interna (0.89) y fiabilidad test-retest $(0.87)$ adecuados. Las relaciones entre autoeficacia académica, metas académicas, autoconcepto académico y rendimiento escolar apoyaron la validez de constructo de la escala. Los modelos de regresión logística para la predicción del rendimiento académico a partir de la autoeficacia y la predicción de ésta a partir del rendimiento apoyaron empíricamente el papel autorregulatorio de la autoeficacia ya que la probabilidad de presentar alta autoeficacia aumenta un $94 \%$ por cada punto que aumenta la nota media, mientras que la probabilidad de presentar éxito académico aumenta un $8 \%$ al incrementarse la autoeficacia académica percibida.
\end{abstract}

Palabras clave: Propiedades psicométricas, autoeficacia académica, metas académicas, autoconcepto académico, rendimiento académico.

Psychometric properties of the Academic Situations Specific Perceived Self-efficacy Scale in a Spanish students sample of Compulsory Secondary Education. The aim of this study was to analyse the psychometric properties of the Academic Situations Specific Perceived Self-efficacy Scale in a 656 Spanish sample of Compulsory Secondary Education students, aged 12 to 16 . Factor analyses revealed that the SPSASS shows a unidimensional structure, with adequate indices of internal consistency (0.89) and test-retest reliability (0.87). Relationships between academic self-efficacy and academic goals, academic selfconcept and academic performance supported the construct validity of the scale. Logistic regression models used to predict academic performance using self-efficacy as predictor, and the prediction of self-efficacy using performance as predictor provided empirical support for the self-regulatory functioning of self-efficacy. It was revealed that the probability of showing high self-efficacy increases in $94 \%$ for each point increase in grade point average, whereas the probability of academic success increases in $8 \%$ when perceived self-efficacy increases.

Key words: Psychometric properties, academic self-efficacy, academic goals, academic self-concept, academic performance.

Correspondencia: Cándido J. Inglés. Área de Psicología Evolutiva y de la Educación. Dpto. de Psicología de la Salud. Universidad Miguel Hernández de Elche. Avda. de la Universidad, s/n. C.P.: 03202 Elche, Alicante (España). E-mail: çingles@umh.es 
La autoeficacia, según Bandura (1997), hace referencia a las capacidades que un individuo percibe que tiene para aprender o llevar a cabo determinadas tareas. Estos juicios que se realizan sobre las propias capacidades se forman a partir del rendimiento existente, el aprendizaje vicario, la información persuasiva proporcionada por otros significativos y los factores fisiológicos derivados de la interpretación de la situación (Schunk y Meece, 2006). A su vez, las creencias de autoeficacia en el ámbito académico influyen en la motivación académica, en la formación del autoconcepto y en el rendimiento escolar (Elliott y Dweck, 1988; Leondari y Gialamas, 2002; Skaalvik, 1997; Smiley y Dweck, 1994). Estas relaciones bidireccionales hacen de las creencias de autoeficacia académica un mecanismo autorregulatorio, según el cual la autoeficacia académica percibida afecta al comportamiento académico y a la elección de las condiciones externas en las que el estudiante actúa y, a su vez, el desarrollo de la autoeficacia académica es influido por estas acciones y condiciones que se dan en el ámbito académico.

\section{Autoeficacia académica y metas académicas en educación secundaria}

La motivación en el contexto académico es un factor influyente en el rendimiento escolar de los adolescentes. Concretamente, los estudios avalan que las orientaciones hacia metas académicas presentadas por los estudiantes tienen un papel importante en el rendimiento escolar (Valle, González-Cabanach, Rodríguez y Piñeiro, 1999).

En el contexto académico, investigaciones previas (Elliott y Dweck, 1988) han destacado que los estudiantes se involucran en las tareas escolares principalmente por dos motivos: (a) aumentar su propia competencia (metas de aprendizaje) y (b) evitar parecer incompetentes frente a otros significativos (metas de rendimiento).

La evidencia empírica previa ha revelado sistemáticamente que la autoeficacia percibida tiene un papel destacado en la motivación y en la elección de metas. En este sentido, diversos autores (Elliott y Dweck, 1988; Smiley y Dweck, 1994) han demostrado la interacción entre capacidad percibida y orientación de meta, encontrando que los individuos con metas de aprendizaje, independientemente de su autoeficacia percibida, eran más persistentes en las tareas tras una experiencia de fracaso, mientras que en los individuos que presentaban una orientación hacia metas de rendimiento se identificaron dos tendencias de actuación diferentes, en función de la autoeficacia percibida. Concretamente, los estudiantes que presentaban una alta autoeficacia percibida se mostraban más persistentes, aunque con menor implicación que los alumnos con metas de aprendizaje, mientras que aquellos otros cuya autoeficacia percibida era baja trataban de evitar la tarea tras la experiencia de fracaso y presentan más emociones negativas. 


\section{Autoeficacia académica y autoconcepto en educación secundaria}

La autoeficacia y el autoconcepto son dos términos estrechamente relacionados entre sí aunque hacen referencia a constructos conceptualmente diferentes. En este sentido, Shalveson, Hubner y Stanton (1976) definen el autoconcepto como el conjunto de autopercepciones que conforman la imagen que una persona tiene de sí misma, formada a partir de factores cognitivos e interacciones sociales a lo largo del ciclo vital. La definición destaca la importancia de la comparación social para la formación del autoconcepto y su relativa estabilidad. En contraposición, la autoeficacia hace referencia a valoraciones específicas del contexto, lo que la hace más fácilmente modificable (Marsh, Craven y McInerney, 2003). De esta forma, mientras la autoeficacia permite establecer juicios sobre tareas o actividades concretas para las que un individuo se siente capacitado, el autoconcepto incluye evaluaciones globales sobre la valía en una determinada área (Zimmerman y Cleary, 2006).

Según Bandura (1997) es posible tener una alta autoeficacia académica sobre una tarea concreta, aunque se tenga un bajo autoconcepto académico general, y obtener buenos resultados académicos. La relación entre estos constructos ha sido evaluada en diversos trabajos en los que se destaca que los juicios de autoeficacia académica correlacionan de forma positiva y significativa con el autoconcepto académico, siendo las magnitudes de estas relaciones elevadas (ej., Skaalvik, 1997).

\section{Autoeficacia académica y rendimiento académico en educación secundaria}

Asimismo, se han hallado relaciones positivas entre la autoeficacia académica percibida para determinadas tareas y el rendimiento académico, siendo esta relación de mayor tamaño cuanto más similares son las medidas en cuanto a la forma, contenido y momento de recogida de datos (Leondari y Gialamas, 2002; Multon, Brown y Lent, 1991; Skaalvik, 1997; Smiley y Dweck, 1994).

En cuanto al papel predictivo del rendimiento académico sobre la percepción de autoeficacia o de ésta sobre el rendimiento, se considera la existencia de una influencia bidireccional y recíproca (Bandura, 1997). En este sentido, estudios previos destacan bien que el rendimiento académico predice la autoeficacia (Breso, Salanova, Martínez, Grau y Agut, 2004), bien que es la percepción de autoeficacia la que predice el rendimiento (Herrera, Ramírez, Roa y Herrera, 2003; Leondari y Gialamas, 2002; Multon et al., 1991). En el presente estudio se pretende analizar la bidireccionalidad de esta relación, estableciéndose la capacidad predictiva de cada uno de los constructos con respecto al otro.

Actualmente, en España existen pocos instrumentos para evaluar la autoeficacia académica. Concretamente, hay una versión española de la Children's Perceived Self-efficacy Scale (Bandura, 1990), realizada por Carrasco y Del Barrio (2002). Sin embargo, ésta sólo incluye una subescala de autoeficacia académica. La 
Escala de Autoeficacia Percibida Específica de Situaciones Académicas (EAPESA; Palenzuela, 1983) permite paliar este problema dado que se trata de un instrumento diseñado para evaluar específicamente autoeficacia académica. Sin embargo, no existen datos acerca de la fiabilidad y validez de esta escala en el primer ciclo de Educación Secundaria Obligatoria (ESO). Además, la estructura interna del instrumento sólo ha sido analizada mediante análisis factorial exploratorio.

Teniendo en cuenta estos hechos, el objetivo de este estudio consiste en examinar las propiedades psicométricas de la EAPESA en una muestra de estudiantes de $1^{\circ}$ a $4^{\circ}$ de E.S.O. Concretamente, se analiza la fiabilidad (consistencia interna y estabilidad temporal) y validez de constructo (estructura factorial y relación con otros constructos tales como rendimiento escolar, orientaciones de metas académicas y autoconcepto). En este sentido, se espera: (a) verificar la estructura unidimensional de la escala, obteniéndose un índice de fiabilidad adecuado $\left(\mathrm{H}_{1}\right)$; (b) hallar relaciones positivas y estadísticamente significativas entre las puntuaciones de autoeficacia y las puntuaciones de metas académicas orientadas al aprendizaje, logro y refuerzo social $\left(\mathrm{H}_{2}\right)$; (c) obtener relaciones positivas y estadísticamente significativas entre las puntuaciones de autoeficacia y las puntuaciones de autoconcepto verbal, matemático y académico general $\left(\mathrm{H}_{3}\right)$; y (d) encontrar relaciones positivas y estadísticamente significativas entre las puntuaciones en autoeficacia y las notas en las asignaturas de Inglés, Lengua Castellana y Matemáticas, así como con la nota media $\left(\mathrm{H}_{4}\right)$.

\section{METODO}

\section{Participantes}

Para la realización de este trabajo se seleccionaron ocho centros de la provincia de Alicante, España, siete públicos y uno privado, mediante muestreo no probabilístico por conveniencia. Una vez seleccionados los centros de estudio se seleccionó aleatoriamente un aula por curso en cada centro, computándose un rango de 78 a 92 sujetos por centro. La muestra reclutada fue de 682 estudiantes de ESO, 281 varones y 375 mujeres, de los que 26 (3.81\%) fueron excluidos por omisiones o errores en sus respuestas, por no obtener por escrito el consentimiento informado de los padres para participar en la investigación o por ser extranjeros con un déficit elevado en el dominio de la lengua española. La muestra final se compuso de 656 adolescentes, con un rango de edad de 12 a 16 años $(M=15.43, D T=1.24)$. La composición étnica de la muestra fue la siguiente: $88.9 \%$ españoles, $6.34 \%$ latinoamericanos, $3.37 \%$ resto Europa, $0.75 \%$ árabes y $0.64 \%$ asiáticos. A través de la prueba $\chi^{2}$ de homogeneidad de la distribución se comprobó la ausencia de diferencias estadísticamente significativas entre los ocho grupos de género x curso $\left(\chi^{2}=7.05, p=0.07\right)$. 


\section{Instrumentos y variables}

Escala de Autoeficacia Percibida Específica de Situaciones Académicas (EAPESA; Palenzuela, 1983). La EAPESA es una escala unifactorial que mide la autoeficacia académica percibida. Consta de 10 ítems valorados mediante una escala de diez puntos, aunque para este estudio la escala de estimación se redujo a 4 puntos $(1=$ nunca; 4 = siempre) por ser más fácil su interpretación para los sujetos de estas edades (12-16 años). A mayor puntuación, mayor autoeficacia académica percibida.

La estructura factorial fue estudiada mediante análisis factorial exploratorio (Palenzuela, 1983). Este análisis indicó que los 10 ítems de la EAPESA saturaron en un único factor, siendo estas saturaciones superiores a 0.63 , excepto para el ítem 9 cuya saturación fue 0.53 .

La matriz de correlaciones inter-ítems reveló correlaciones adecuadas para todos los ítems, variando desde 0.30 a 0.70 . Las correlaciones ítem-total también fueron adecuadas, variando desde 0.45 a 0.78 . El coeficiente alfa de Cronbach para la escala fue 0.91. La fiabilidad test-retest, para un intervalo temporal de 10 semanas, fue 0.92. Las relaciones encontradas entre la EAPESA y otros cuestionarios conceptualmente afines (atribuciones académicas, motivación escolar, ansiedad ante la evaluación académica y autoestima) apoyaron su validez de constructo (Palenzuela, 1983).

Cuestionario de Evaluación de Metas Académicas (CEMA, Achievement Goal Tendencies Questionnaire; Hayamizu y Weiner, 1991). El CEMA es un autoinforme compuesto por 20 ítems diseñado para medir tres tendencias u orientaciones de metas, identificadas mediante análisis factorial: (a) Metas de Aprendizaje (MA), indican la tendencia de los estudiantes por aprender con el propósito de adquirir conocimientos y aumentar su competencia; (b) Metas de Logro (ML), reflejan la tendencia de los estudiantes por aprender con la intención de obtener buenas notas en los exámenes y avanzar en los estudios; y (c) Metas de Refuerzo Social (MRS), indican la tendencia de los estudiantes por aprender para obtener juicios de aprobación y evitar el rechazo de padres y profesores. Cada ítem se responde mediante una escala de 5 puntos $(1=$ nunca $; 5$ = siempre $)$.

Las propiedades psicométricas del CEMA han sido analizadas en estudiantes españoles de educación primaria, secundaria y educación superior (González-Pienda et al., 2000; Inglés et al., 2009; Navas, González y Torregrosa, 2002; Valle, GonzálezCabanach, Cuevas y Núñez, 1997), hallándose niveles aceptables de fiabilidad y validez de constructo.

Cuestionario de Autodescripción-II (SDQ-II, Self-Description QuestionnaireII; Marsh, 1992). El SDQ-II es una medida de autoinforme diseñada para evaluar el autoconcepto en adolescentes de 12 a 18 años. Contiene 102 ítems distribuidos en 11 escalas, tres académicas (Matemáticas, Verbal y Académica General) y siete no académicas (Habilidades Físicas, Apariencia Física, Relaciones con el Sexo Opuesto, 
Relaciones con el Mismo Sexo, Relación con los Padres, Sinceridad-Veracidad y Estabilidad Emocional), incluyendo, además, una escala de Autoestima y una puntuación total de autoconcepto general. Los ítems se puntúan según una escala de respuesta de 6 puntos $(1=$ falso; 6 =verdadero $)$.

Las investigaciones realizadas sobre este cuestionario en población adolescente española (García-Fernández et al., 2006) aportaron resultados que replicaron la estructura original del instrumento y apoyaron su validez de constructo.

Para los objetivos de este estudio únicamente se tuvieron en cuenta las escalas de autoconcepto académico: Matemáticas, Verbal y Académica General, cuyos coeficientes alfa de Cronbach fueron: 0.92 (Matemáticas), 0.84 (Verbal) y 0.90 (Académico General).

Rendimiento académico. El rendimiento académico, definido como el nivel de logro que el estudiante puede obtener en una asignatura en particular o en la media de un conjunto de ellas, fue evaluado a través de las notas obtenidas por los estudiantes en las asignaturas de Lengua Castellana, Matemáticas e Inglés, así como la media de todas las asignaturas.

\section{Procedimiento}

En primer lugar, se llevó a cabo una entrevista con los directores y orientadores de los centros participantes, con el fin de exponer los objetivos de la investigación, describir los instrumentos de evaluación, solicitar permiso y promover su colaboración. Los autoinformes fueron contestados de forma colectiva, voluntaria y anónima en el aula, asignando previamente un número de identificación a las hojas de respuesta entregadas a cada sujeto, las cuales fueron posteriormente corregidas mediante ordenador.

Se solicitó a los participantes que cumplimentaran los datos de identificación (género, edad, curso) y se leyeron en voz alta las instrucciones, haciendo énfasis en la importancia de no dejar respuestas sin contestar. Los investigadores permanecieron en el aula durante la aplicación de las pruebas con tal de resolver las posibles dudas que aparecieran y verificar la correcta cumplimentación de las respuestas y datos de identificación. El orden de presentación de las pruebas se estableció aleatoriamente para cada grupo de estudiantes.

\section{RESULTADOS}

\section{Estructura interna; Análisis factorial exploratorio}

Para analizar la estructura factorial de la EAPESA se empleó el análisis factorial de componentes principales y el análisis factorial confirmatorio. 
Antes de realizar el análisis de componentes principales se analizó la adecuación muestral mediante la prueba Kaiser-Meyer-Olkin (KMO) obteniéndose valores satisfactorios $(K M O=0.93, p<0.01)$. Asimismo, el Test de Esfericidad de Barlett permitió corroborar la adecuada correlación entre los ítems $(p=0.00)$.

$\mathrm{El}$ análisis de componentes principales mostró que los 10 ítems se agruparon en un solo factor, explicando el $51.47 \%$ de la varianza. La matriz factorial presentó cargas superiores a 0.30 para todos los ítems.

\section{Estructura interna; Análisis Factorial Confirmatorio}

Se evaluaron dos modelos factoriales: el modelo nulo $\left(\mathrm{M}_{0}\right)$, que asume la máxima independencia entre ítems, es decir, la no existencia de una estructura factorial, y el modelo de un factor $\left(M_{1}\right)$ que mantiene la existencia de un único factor explicativo.

Para evaluar la adecuación de los modelos se utilizaron cuatro índices de bondad de ajuste: la raíz residual estandarizada cuadrática media (SRMR), el índice de bondad de ajuste (GFI), el índice de bondad de ajuste corregido (AGFI) y la raíz media cuadrática del error de aproximación (RMSEA). Un ajuste adecuado del modelo está determinado por valores menores de 0.08 en SRMR, valores en GFI iguales o superiores a 0.90 , valores iguales o superiores a 0.85 en AGFI y valores inferiores a 0.06 en RMSEA. Tal y como mostraron los resultados, el modelo de un factor (M1) presentó un ajuste adecuado (véase tabla 1 ).

Tabla 1. Análisis factorial confirmatorio

\begin{tabular}{lccccccc}
\hline Modelo & $\chi^{2}$ & $g l$ & $p$ & SRMR & RMSEA & GFI & AGFI \\
\hline Nulo (Mo) & 2856.7 & 45 & 0.00 & 0.303 & 0.309 & 0.34 & 0.19 \\
Un factor $\left(\mathrm{M}_{1}\right)$ & 141.6 & 35 & 0.00 & 0.025 & 0.051 & 0.96 & 0.93 \\
\hline
\end{tabular}

\section{Fiabilidad}

La consistencia interna fue hallada mediante el coeficiente de consistencia interna alfa de Cronbach y la fiabilidad test-retest fue calculada a través del coeficiente de correlación producto-momento de Pearson.

El coeficiente de consistencia interna de la escala fue 0.89 . En cuanto a la aportación de cada ítem a la fiabilidad de la escala, todos ellos contribuyeron a aumentar la fiabilidad de la prueba dado que su eliminación implicaba una disminución del coeficiente alfa. La fiabilidad test-retest, para un intervalo de 3 semanas, fue 0.87 .

Las correlaciones ítem-total también fueron elevadas para todos los ítems, oscilando entre 0.37 y 0.71 . Los coeficientes de correlación inter-ítems variaron de 0.33 a 0.60 . 


\section{Correlación entre autoeficacia académica y metas académicas}

Para analizar la relación entre autoeficacia académica y metas académicas, autoconcepto y rendimiento escolar se empleó el coeficiente de correlación productomomento de Pearson. La interpretación de este coeficiente se realiza siguiendo los criterios de Cohen (1988), quien sugiere que valores iguales o mayores que $0.10 \mathrm{e}$ inferiores a 0.30 indican una relación de pequeña magnitud, valores entre 0.30 y 0.49 indican una magnitud media y valores iguales o mayores de 0.50 indican un tamaño del efecto elevado.

Los resultados indicaron que existía una relación estadísticamente significativa, positiva y moderada (Cohen, 1988) entre autoeficacia académica y metas orientadas al logro $(r=0.48)$. Sin embargo, las correlaciones entre autoeficacia académica y las metas orientadas al aprendizaje y al refuerzo social, aunque también fueron positivas, no resultaron estadísticamente significativas (véase tabla 2).

Tabla 2. Correlaciones entre autoeficacia académica, metas académicas, autoconcepto académico y rendimiento académico

\begin{tabular}{lcc}
\hline Escalas & \multicolumn{2}{c}{ Autoeficacia percibida (EAPSA) } \\
\hline & $r$ & $p$ \\
\hline Metas académicas (CEMA) & & 0.09 \\
\hline Metas de aprendizaje & 0.22 & 0.24 \\
Metas de Refuerzo social & 0.18 & 0.00 \\
Metas de logro & 0.48 & 0.00 \\
\hline Autoconcepto académico (SDQ-II) & & 0.00 \\
Matemático & & 0.00 \\
Verbal & 0.31 & 0.27 \\
Académico general & 0.44 & 0.00 \\
Rendimiento académico & & 0.00 \\
& & 0.00 \\
Castellano & 0.38 & 0.00 \\
Matemáticas & 0.35 & 0.28 \\
Rendimiento medio & 0.36 & \\
\hline
\end{tabular}

\section{Correlación entre autoeficacia académica y autoconcepto}

Los resultados mostraron la existencia de una correlación positiva y estadísticamente significativa entre las puntuaciones obtenidas en autoeficacia académica y las tres subescalas de autoconcepto académico del SDQ-II, siendo esta correlación moderada respecto a la escala de autoconcepto Académica General y baja respecto a las escalas Matemática y Verbal (véase tabla 2). 


\section{Correlación entre autoeficacia académica y rendimiento académico}

Los resultados mostraron una correlación positiva, moderada y estadísticamente significativa entre la puntuación de autoeficacia académica y la nota en las asignaturas de Lengua Castellana e Inglés, así como Rendimiento medio (media de todas las asignaturas). Sin embargo, esta correlación presentó un tamaño del efecto pequeño en relación con las calificaciones en Matemáticas (véase tabla 2).

\section{Autoeficacia académica y éxito académico: ¿Relación bidireccional?}

El establecimiento de modelos que permitiesen pronosticar el rendimiento académico medio y la autoeficacia académica percibida se llevó a cabo mediante análisis de regresión logística, siguiendo el procedimiento de regresión por pasos hacia adelante basado en el estadístico de Wald. Para realizar estos análisis fue necesario dicotomizar las variables criterio, definiéndose el rendimiento académico medio como éxito (media igual o mayor a 5 puntos) y fracaso (media inferior a 5 puntos) y la autoeficacia como alta autoeficacia (puntuaciones iguales o superiores a 31.62; equivalente a la media más una desviación típica) y baja autoeficacia (puntuaciones iguales o inferiores a 19.54; equivalente a la media menos una desviación típica).

El establecimiento del modelo que permitía pronosticar la autoeficacia académica a partir de la nota media de los estudiantes en todas las asignaturas reveló, teniendo en cuenta el valor de la odds ratio (OR) obtenida, que la probabilidad de presentar altas puntuaciones en autoeficacia es 1.94 veces mayor por cada punto que incrementa la nota media de los estudiantes (véase tabla 3). Es decir, la probabilidad de presentar alta autoeficacia aumentaba un $94 \%$.

Igualmente, la regresión logística binaria también se utilizó para establecer una ecuación de predicción del éxito académico (media igual o mayor a 5 puntos) empleando como variable predictora la autoeficacia académica. En este caso, el valor de la OR reveló que la probabilidad de presentar una nota media igual o mayor a cinco era 1.08 veces mayor por cada punto que se incrementaba la puntuación en autoeficacia académica, por lo que la probabilidad de aprobar (éxito académico) aumentaba un $8 \%$ (véase tabla 3).

Tabla 3. Regresiones logísticas

\begin{tabular}{|c|c|c|c|c|c|c|}
\hline \multicolumn{7}{|c|}{ Regresión logística para la probabilidad de alta autoeficacia académica } \\
\hline & $\mathrm{B}$ & E.T. & Wald & $p$ & OR & IC $95 \%$ \\
\hline Rendimiento medio & 0.66 & 0.15 & 19.12 & 0.00 & 1.94 & $1.44-2.61$ \\
\hline Constante & -3.83 & 0.94 & 16.60 & 0.00 & 0.02 & \\
\hline \multicolumn{7}{|c|}{ Regresión logística para la probabilidad de éxito académico } \\
\hline & $\mathrm{B}$ & E.T. & Wald & $p$ & OR & IC 95\% \\
\hline Autoeficacia & 0.08 & 0.02 & 10.40 & 0.00 & 1.08 & $1.03-1.14$ \\
\hline Constante & -1.00 & 0.64 & 2.47 & 0.12 & 0.37 & \\
\hline
\end{tabular}




\section{DISCUSION}

El objetivo del presente estudio fue analizar las propiedades psicométricas de la Escala de Autoeficacia Percibida en Situaciones Académicas, utilizando para ello una muestra de estudiantes de ESO. Como se esperaba, los análisis factoriales confirmaron la unidimensionalidad de la EAPSA, coincidiendo con los resultados hallados por el autor original. Asimismo, los resultados obtenidos indicaron una adecuada fiabilidad del instrumento, mostrando índices de consistencia interna y fiabilidad test-retest elevados y similares a los informados por Palenzuela (1983).

La segunda hipótesis de este trabajo planteaba que la autoeficacia académica correlacionaría positiva y significativamente con las metas académicas orientadas al aprendizaje, al logro y al refuerzo social. En este sentido se observó que aunque la percepción de autoeficacia en el ámbito académico correlacionó positivamente con las tres orientaciones de meta, únicamente la relación con metas de rendimiento o logro resultó estadísticamente significativa, indicando que mayores puntuaciones en autoeficacia académica se relacionaban con una mayor tendencia a estudiar para sacar buenas notas y avanzar en los estudios. El que no se hayan encontrado relaciones significativas entre autoeficacia académica percibida y las orientaciones de meta al aprendizaje y al refuerzo social podría deberse al contenido de las escalas administradas en este estudio. Al realizar un análisis pormenorizado del contenido de los ítems se observa que la escala de autoeficacia hace especial referencia a la eficacia percibida para conseguir logros académicos (por ejemplo, "estoy preparado para conseguir muchos éxitos académicos o para obtener un buen expediente académico”) más que a la eficacia por mejorar en los conocimientos (relacionada con las metas de aprendizaje).

En tercer lugar, en el presente estudio se hipotetizaba que existiría una relación significativa y positiva entre la percepción de autoeficacia académica y el autoconcepto académico general, matemático y verbal. Los resultados confirmaron esta hipótesis, apareciendo las relaciones de mayor tamaño entre autoeficacia académica y autoconcepto académico general. El que esta relación fuera la de mayor tamaño se debe a que la escala de autoeficacia percibida empleada en este estudio, pese a ser específica del ámbito académico, no hace referencia a percepciones de autoeficacia en dominios académicos concretos (por ejemplo, matemáticas o lengua castellana). Por tanto, era razonable esperar que las relaciones con las escalas concretas de autoconcepto académico (matemático y verbal) fueran menores.

Las relaciones entre las notas medias en las diferentes asignaturas y la autoeficacia escolar percibida apoyaron la última hipótesis. Como ha sido indicado, estas relaciones fueron de magnitud pequeña o moderada debido a que la prueba que evalúa la autoeficacia no hace referencia a dominios académicos específicos. Asimismo, la relación entre la puntuación media de todas las asignaturas y la autoeficacia percibida 
presentó una magnitud moderada. Estos datos coincidieron con los encontrados por Multon et al. (1991) quienes constataron, a través de un estudio meta-analítico en el que se evaluaba la relación entre autoeficacia y rendimiento académico, que la media de los coeficientes de correlación entre ambas variables fue de 0.32 , apareciendo coeficientes más altos en aquellos estudios en los que las medidas de autoeficacia eran específicas de la tarea evaluada.

Los resultados de este estudio revelaron que la autoeficacia percibida pronosticaba el éxito académico y éste pronosticaba, a su vez, una elevada autoeficacia académica percibida. Concretamente, la probabilidad de presentar alta autoeficacia aumentaba un $94 \%$ por cada punto que se incrementaba la nota media, mientras que la probabilidad de presentar éxito académico aumentaba un $8 \%$ al incrementarse la autoeficacia académica. Las investigaciones previas señalan la importancia de la autoeficacia académica percibida en la predicción del aprendizaje y logro académico, siendo ésta una variable de peso en la explicación del rendimiento (Herrera et al., 2003; Leondari y Gialamas, 2002; Multon et al., 1991). En el presente estudio la autoeficacia presentó un papel modesto en la predicción del rendimiento escolar, lo que podía explicarse por la forma de categorizar el rendimiento. En este sentido, quizá resultaría interesante distinguir, por ejemplo, el alto rendimiento como un rendimiento destacado (nota media igual o superior a 7 puntos). De esta forma se podría obtener una visión más ajustada sobre la influencia de la autoeficacia en el rendimiento académico. Asimismo, el papel predictor ejercido por el rendimiento académico sobre la autoeficacia corroboraba los resultados obtenidos en investigaciones previas (Breso et al., 2004) confirmando la influencia de los comportamientos sobre las propias percepciones de eficacia. Estos datos proporcionan apoyo empírico a la teoría de Bandura respecto al papel autorregulatorio de la autoeficacia, mostrándose predictora del rendimiento académico y siendo influida por el mismo. Sin embargo, cabe destacar que el carácter transversal de esta investigación impide establecer relaciones causales entre autoeficacia y rendimiento académico, aspecto que debería ser abordado en próximos estudios utilizando la metodología de ecuaciones estructurales. Además, resultaría interesante tener en cuenta en los modelos otros factores que pueden estar influyendo en la relación entre autoeficacia y rendimiento, tales como las atribuciones de metas (Elliott y Dweck, 1988; Smiley y Dweck, 1994), el autoconcepto (Skaalvik, 1997), la edad (Multon et al., 1991) y los conocimientos y habilidades previas (Schunk y Meece, 2006).

En resumen, este estudio aporta datos que permiten apoyar la validez de constructo de la Escala de Autoeficacia Percibida Específica de Situaciones Académicas, destacando su unidimensionalidad, fiabilidad y relación con constructos afines en estudiantes de ESO, ampliando de esta forma la posibilidad de usar la escala no sólo con población universitaria y adolescente sino también preadolescente. Asimismo, este estudio demuestra como la aplicación de la teoría de la autoeficacia de Bandura en 
el ámbito educativo permite comprobar que los estudiantes con altas expectativas de autoeficacia presentan una elevada motivación de logro, tienen una percepción más positiva de sí mismos en el ámbito escolar y obtienen mejores resultados académicos.

Las investigaciones previas han puesto de manifiesto que la implicación activa del sujeto en el proceso de aprendizaje aumenta cuando se siente autocompetente, es decir, cuando confía en sus propias capacidades y tiene altas expectativas de autoeficacia (Elliott y Dweck, 1988; Schunk y Meece, 2006; Smiley y Dweck, 1994). Asimismo, cabe señalar que la autoeficacia percibida en el ámbito académico es un aspecto destacable no únicamente desde el punto de vista del estudiante, sino que, tal y como muestran estudios recientes (Pacheco, García y Díez, 2009), la autoeficacia percibida por los profesores sobre su propio desempeño también influye en la forma de estructurar su docencia en la enseñanza de la escritura, en relación a los materiales y textos utilizados en sus clases.

En definitiva, tal y como señalan los resultados de este estudio, la mejora en las expectativas de autoeficacia puede incrementar el autoconcepto escolar, la motivación y el rendimiento en tareas de aprendizaje, por lo que parece necesario que los esfuerzos educativos se dirijan hacia el aumento de los sentimientos de autovaloración y competencia de los estudiantes, con el fin de mejorar su funcionamiento en las tareas de aprendizaje.

\section{Agradecimientos}

Este trabajo ha sido realizado a través del Proyecto SEJ 2004-07311/EDUC perteneciente al Plan Nacional de Investigación Científica, Desarrollo e Innovación Tecnológica del Ministerio de Educación y Ciencia concedido al segundo autor y de una Beca Predoctoral de la Fundación Cajamurcia concedida a la tercera autora.

\section{REFERENCIAS}

Bandura, A. (1997). Self-efficacy: The exercise of control. New York: Freeman.

Bandura, A. (1990). Multidimensional scales of perceived academic efficacy. Stanford, CA: Stanford University.

Breso, E., Salanova, M., Martínez, I.M., Grau, R. y Agut, S. (2004). Éxito académico y expectativas de éxito: el rol mediador de la autoeficacia académica. En M. Salanova, R. Grau, I.M. Martínez, S. Llorens y M. García-Renedo (Eds.), Nuevos horizontes en la investigación sobre la autoeficacia (pp. 237-243). Castelló de la Plana: Publicaciones de la Universidad Jaume I, D. L.

Carrasco, M.A. y Del Barrio, M.V. (2002). Evaluación de la autoeficacia en niños y adolescentes. Psicothema, 14(2), 323-332.

Cohen, J. (1988). Statistical power analysis for the behavioral sciences (2nd Ed.). Hillsdale, NJ, USA: Erlbaum.

Elliott, E.S. \& Dweck, C.S. (1988). Goals: An approach to motivation and achievement. Journal of Personality and Social Psychology, 54(1), 5-12. 
García-Fernández, J.M., Torregrosa, M.S., Inglés, C.J., Ruiz-Esteban, C., Pastor, Y., MartínezZaragoza, F., Espada, J.P. \& Delgado, B. (2006). Analysis of the self-concept dimensionality in a Spanish sample of Secondary School students. Trabajo presentado en el 26th Congress of Applied Psychology, Julio, Atenas.

González-Pienda, J.A., Núñez, J.C., González-Pumariega, S., Álvarez, L., Roces, C., García, M., González, P., González-Cabanach, R.G. y Valle, A. (2000). Autoconcepto, proceso de atribución causal y metas académicas en niños con y sin dificultades de aprendizaje. Psicothema, 12(4), 548-556.

Hayamizu, T. y Weiner, B. (1991). A test of Dweck's model of achievement goals as related to perceptions of ability. Journal of Experimental Education, 59, 226-234.

Herrera, F., Ramírez, M.I., Roa, J.M. y Herrera, I. (2003). Tratamientos de las creencias motivacionales en contextos educativos pluriculturales. Revista Iberoamericana de Educación. http://www.rieoei.org/investigacion/625Herrera.PDF[Consulta: mayo 2009].

Inglés, C.J., García-Fernández, J.M. Castejón, J.L., Valle, A., Delgado, B. y Marzo, J.C. (2009). Reliability and validity evidence of scores on the Achievement Goal Tendencies Questionnaire in a sample of Spanish students of compulsory secondary education. Psychology in the Schools, 46(10), 1048-1060.

Leondari, A. \& Gialamas, V. (2002). Implicit theories, goal orientations, and perceived competence: impact on students' achievement behavior. Psychology in the Schools, 39(3), 279-291.

Marsh, H.W. (1992). SDQ II: Manual. Sydney, Australia: Self Research Centre, University of Western Sydney.

Marsh, H.W., Craven, R.G. \& McInerney, D.M. (2003). International advances in self research. En H.W. Marsh, R.G., Craven \& D.M. McInerney (Eds.), International advances in self research (pp. 3-14). Greenwich, CT: Information Age Publishing.

Multon, K.D., Brown, S.D. \& Lent, R.W. (1991). Relation of self-efficacy beliefs to academic outcomes: A meta-analytic investigation. Journal of Counselling Psychology, 38(1), 3038.

Navas, L., González, C. y Torregrosa, G. (2002). Metas de aprendizaje: Un análisis transversal de las estructuras factoriales que presentan. Revista de Psicología General y Aplicada, 55(3), 553-564.

Pacheco, D.I., García, J.N. y Díez, C. (2009). Autoeficacia, enfoque y papel de la práctica de los maestros en la enseñanza de la escritura. European Journal of Education and Psychology, 2(1), 5-23.

Palenzuela, D. (1983). Construcción y validación de una escala de autoeficacia percibida específica de situaciones académicas. Análisis y Modificación de Conducta, 9(21), 185219.

Schunk, D.H. \& Meece, J.L. (2006). Self-efficacy development in adolescence. En F. Pajares \& T. Urdan (Eds.), Self-efficacy beliefs of adolescence (pp. 71-96). USA: Information Age Publishing.

Shavelson, J., Hubner, J.J. \& Stanton, G.C. (1976). Self-concept: validation of construct interpretations. Review of Educational Research, 46(3), 407-442.

Skaalvik, E.M. (1997). Self-enhancing and self-defeating ego orientation: Relations with task and avoidance orientation, achievement, self-perceptions and anxiety. Journal of Educational Psychology, 89(1), 71-81.

Smiley, P.A. \& Dweck, C.S. (1994). Individual differences in achievement goals among young children. Child Development, 65(6), 1723-1743.

Valle, A., González-Cabanach, R., Cuevas, L.M. y Núñez, J.C. (1997). Patrones motivacionales en estudiantes universitarios. Revista Investigación Educativa, 15(1), 125-146. 
Valle, A., González-Cabanach, R., Rodríguez, S. y Piñeiro, I. (1999). Atribuciones causales, autoconcepto y motivación en estudiantes con alto y bajo rendimiento académico. Revista Española de Pedagogía, 214, 525-545.

Zimmerman, B.J. \& Cleary, T.J. (2006), Adolescents' development of personal agency. En F. Pajares \& T. Urdan (Eds.), Self-efficacy beliefs of adolescence (pp. 71-96). USA: Information Age Publishing.

Recibido: 13 de junio de 2009 Recepción Modificaciones: 20 de noviembre de 2009 Aceptado: 25 de noviembre de 2009 